\title{
ICOS protects against mortality from acute lung injury through activation of IL-5 $5^{+} \mathrm{ILC} 2 \mathrm{~S}$
}

\author{
CL Hrusch $^{1}$, ST Manns ${ }^{1}$, D Bryazka ${ }^{1}$, J Casaos ${ }^{1}$, CA Bonham ${ }^{1}$, MR Jaffery ${ }^{1}$, KM Blaine ${ }^{1}$, KAM Mills $^{1}$, PA Verhoef ${ }^{1}$, \\ AO Adegunsoye ${ }^{1}$, JW Williams ${ }^{1}$, MY Tjota ${ }^{1}$, TV Moore ${ }^{1}$, ME Strek ${ }^{1}$, I Noth ${ }^{1}$ and AI Sperling ${ }^{1,2}$
}

Idiopathic pulmonary fibrosis (IPF) is a progressive lung disease causing irreversible lung scarring and loss of pulmonary function. IPF Patients suffer from a high rate of pulmonary infections and acute exacerbations of disease that further contribute to pulmonary decline. Low expression of the inducible T-cell costimulatory molecule (ICOS) in peripheral blood mononuclear cells predicts decreased survival of IPF patients, but the mechanisms by which ICOS protects are unclear. Using a model of bleomycin-induced lung injury and fibrosis, we now demonstrate that ICOS expression enhances survival from lung injury rather than regulating fibrogenesis. Of ICOS-expressing cells, type 2 innate lymphocytes (ILC2s) are the first to respond to bleomycin-induced injury, and this expansion is ICOS dependent. Interestingly, a similar decrease in ICOS ${ }^{+}$ILCs was found in lung tissue from IPF patients. Interleukin (IL)-5, produced primarily by ILC2s, was significantly reduced after lung injury in ICOS ${ }^{-1-}$ mice, and strikingly, treatment with IL-5 protected both ICOS ${ }^{-1-}$ and wild-type mice from mortality. These results imply that low ICOS expression and decreased lung ILC2s in IPF patients may contribute to poor recovery from infections and acute exacerbation and that IL-5 treatment may be a novel therapeutic strategy to overcome these defects and protect against lung injury.

\section{INTRODUCTION}

Nearly 50,000 Americans are diagnosed each year with idiopathic pulmonary fibrosis (IPF), a progressive disease with a median survival of 3-5 years. The etiology of IPF is thought to be due to repeated pulmonary epithelial injury, aberrant tissue repair, and excessive accumulation of extracellular matrix in the lung parenchyma. ${ }^{1-3}$ Although two recent Food and Drug Administration-approved treatments slow the loss of pulmonary function by targeting fibrogenesis, no treatments exist to reverse disease course, and lung transplant is often indicated as a final treatment option. The role of the immune system in IPF remains controversial. Recent clinical studies found that suppressing immune function in IPF patients was associated with increased mortality, suggesting a beneficial role for the immune system. ${ }^{4}$ Further, during acute exacerbations of IPF, steroid treatment can adversely affect IPF patients and lead to decreased survival. ${ }^{5}$ We recently reported that $\mathrm{T}$ cells are increased in the lungs of IPF patients and that subsets expressing specific chemokine receptors are associated with improved lung function. ${ }^{6}$ Gene expression profiles in peripheral blood mononuclear cells from IPF patients also have implicated a role for $\mathrm{T}$ cells in protection. ${ }^{7}$ In particular, low expression of the inducible $\mathrm{T}$-cell costimulatory molecule (ICOS) in peripheral blood mononuclear cells predicted decreased long-term transplant-free survival. Thus a role for the immune system in IPF etiology or pathology is emerging.

ICOS was first identified as a T helper type 2 cell-specific costimulatory receptor, ${ }^{8}$ and $\mathrm{ICOS}^{-1-}$ mice have defects in type 2 inflammation. ${ }^{9-11}$ ICOS $^{+}$cells resident in the lung include type 2 innate lymphocytes (ILC2s), invariant natural killer T cells (iNKTs), and CD4 T cells. ${ }^{12-14}$ These cells produce the cytokines interleukin (IL)-4, IL-5, and IL-13 associated with atopic diseases. Studies utilizing bleomycin to induce lung fibrosis in mice found that both type 17 and type 2 responses are associated with transforming growth factor- $\beta$ production and subsequent fibrosis. ${ }^{15}$ The type 17 immune response is characterized by neutrophilia that leads to lung damage from reactive oxygen species, proteinases, and matrix metalloproteinases. The type 2 immune response in pulmonary fibrosis is

${ }^{1}$ Department of Medicine, Section of Pulmonary and Critical Care, University of Chicago, Chicago, Illinois, USA and ${ }^{2}$ Committee on Immunology, University of Chicago, Chicago, Illlinois, USA. Correspondence: Al Sperling (asperlin@uchicago.edu) 
associated with M2 macrophage polarization, fibroblast activation, and collagen deposition. As $\mathrm{ICOS}^{+}$lymphocytes are associated with type 2 responses and fibrogenesis, why low ICOS expression in IPF patients is associated with worse outcomes is unclear.

Using bleomycin to model acute lung injury-induced fibrosis, we now demonstrate that ICOS expression is required for protecting against lung injury-induced mortality but does not regulate fibrogenesis. Further, ICOS expression is required for IL-5 production in the lungs post-injury, and this IL-5 is produced primarily by ILC2s. Exogenous IL-5 delivered to the airways of ICOS ${ }^{-1-}$ mice was sufficient to protect from lung injury-induced death, and importantly, IL-5 treatment was also effective in reducing mortality in wild-type (WT) mice that received twofold higher doses of bleomycin. Notably, lung injury-induced edema was greatly reduced when $\mathrm{ICOS}^{-1-}$ mice were treated with exogenous IL-5. These findings suggest a mechanism whereby IL-5 production from ILC2s protects mice from lung injury. As acute exacerbations of IPF are associated with lung injury, IL-5 may be a novel therapeutic target for preventing exacerbation-induced decline in IPF patients.

\section{RESULTS}

ICOS-deficient mice have an impaired ability to survive the early lung injury phase induced by bleomycin challenge

To address the role of ICOS in pulmonary fibrosis, we compared ICOS $^{-1-}$ mice to WT mice using a model of bleomycin-induced lung injury. Strikingly, compared with WT mice, $\mathrm{ICOS}^{-1-}$ mice exhibited dramatically reduced survival and increased loss of body weight (Figure 1a,b). When only the $\mathrm{ICOS}^{-1-}$ mice that survived until day 18 after challenge were analyzed, they also showed increased weight loss compared with WT mice (Figure 1c), yet had no difference in lung fibrosis or collagen deposition (Figure 1d,e). Mice heterozygous for ICOS were protected from weight loss despite their intermediate ICOS expression on lung lymphocyte populations (see Supplementary Figure S1 online).

We suspected that early mortality in $\mathrm{ICOS}^{-1-}$ mice was caused by increased acute lung injury, as these mice lost weight within 5 days of challenge, whereas WT mice exhibited little weight loss. We observed that $\mathrm{ICOS}^{-1-}$ mice had increased red blood cells (RBCs) in the airways on day 5 (Figure 1f,g), indicating greater lung edema, a hallmark of lung injury. Interestingly, no difference was observed in lung inflammation in histology sections or in bronchoalveolar lavage (BAL) protein levels (Figure 1h,i). Consistent with our finding of increased edema in $\mathrm{ICOS}^{-1-}$ mice, increased pulmonary vascular permeability was also observed (Figure 1j). Finally, lung neutrophil numbers were significantly increased when measured by flow cytometry (Figure 1k). Together, these changes indicate a specific defect in $\mathrm{ICOS}^{-1-}$ mice that leads to enhanced vascular leakage and neutrophilia after bleomycin challenge.

To determine whether ICOS had an essential role in other lung injury models, PR8 influenza infection and lipopolysaccharide airway challenge were examined in the $\mathrm{ICOS}^{-1}$ mice.
Similar to the bleomycin model, ICOS ${ }^{-1-}$ mice had increased weight loss and mortality in response to lung injury induced by influenza infection, with the majority of mice succumbing by day 10 postinfection (see Supplementary Figure S2). Similarly, lipopolysaccharide instillation in the airways led to increased weight loss in $\mathrm{ICOS}^{-1-}$ mice compared with WT at $48 \mathrm{~h}$ (see Supplementary Figure S3). Together, these data suggest an important role for ICOS in protection of mice in three separate lung injury models but not a direct role for ICOS in alleviating collagen deposition and fibrosis.

\section{Innate ICOS-expressing cells are reduced in the lungs of ICOS $^{-1-}$ mice after bleomycin challenge}

To determine the mechanisms by which ICOS protects from early mortality, we first examined cellular infiltrates in the lung tissue at 3 days post-challenge. Total isolated cell numbers were not different in the mouse strains, nor were the percentage of alveolar macrophages, neutrophils, or eosinophils at this early time point (Figure $2 \mathbf{a}$ ), although neutrophils were increased in ICOS $^{-1-}$ mice by day 5 (Figure 1k). Next we assessed the contributions of ICOS-expressing cell types. As previously reported, ${ }^{12,13}$ we found that untreated ICOS $^{-1-}$ mice had fewer ILC2s and iNKTs in the lungs (see Supplementary Figure S4). In WT mice, bleomycin induced an expansion of ILC2 3 days after challenge, but ILC2s from ICOS $^{-1-}$ mice failed to expand until day 7 (Figure $\mathbf{2 b}, \mathbf{c}$ ). In contrast to ILC2s, the proportion of iNKTs did not increase in $\mathrm{ICOS}^{+/+}$mice until day 7 after challenge, an increase not observed in ICOS $^{-1-}$ mice (Figure 2d). CD4 T cells were found at a similar percentage and number in both mouse strains (Figure 2e), although the proportion identified as FoxP3 + regulatory T cells (Tregs) was only increased in WT mice (see Supplementary Figure S5A). Surprisingly, bleomycin also induced increased ICOS expression on ILC2 over time but not on iNKTs or CD4 T cells (Figure $2 \mathbf{f}$ and see Supplementary Figure S5B). In addition, we found an increase in ICOSL on alveolar macrophages and monocyte-derived macrophages over time in both WT and ICOS $^{-1-}$ mice, suggesting a role for macrophages in early ICOS signaling (Supplementary Fig 6). Thus ICOS-dependent ILC2s, and not iNKTs or T cells, are the first ICOS-expressing cells to expand during the early inflammatory phase after bleomycin challenge. Further, the failure of iNKTs and Tregs to expand at day 7 after lung injury may contribute to the poor recovery of the $\mathrm{ICOS}^{-1-}$ mice.

To determine whether ICOS-expressing innate cells might contribute to human disease, we obtained lung tissue from nine IPF patients at the time of lung transplant. Control lungs were obtained through the Gift of Hope Organ Bank of Illinois from organ donors with no known lung disease. A group of 13 control lungs were selected to match the IPF patients based on age, sex, race, and smoking history (Table 1). As previously shown by others, we found that IPF lungs had significantly increased eosinophils (measured by histological counts) compared with the control lungs $(39.25 \pm 29.93$ eos per field in IPF patients vs. $2.1 \pm 1.7$ in controls; $P<0.0001$ ).

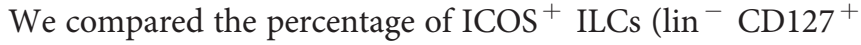



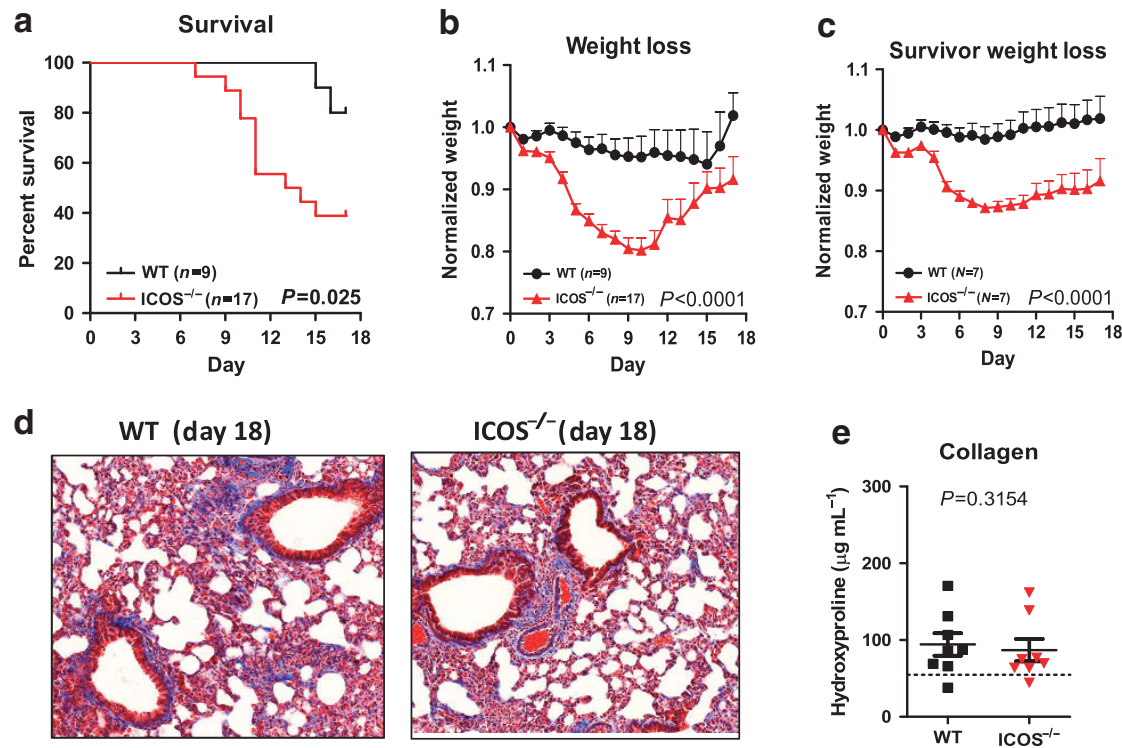

f
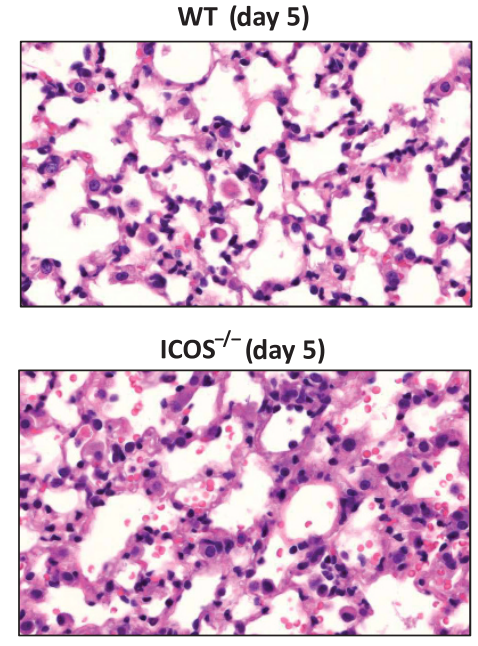

g

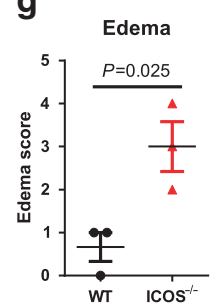

h

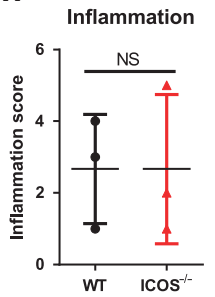

i

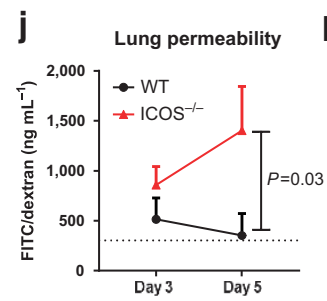

k

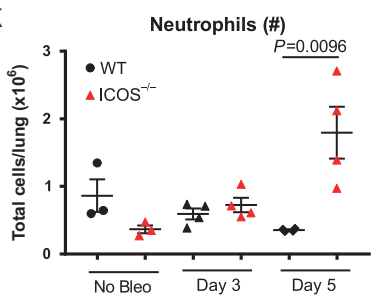

Figure 1 Bleomycin-challenged ICOS ${ }^{-1-}$ mice exhibit similar lung collagen deposition compared with wild-type (WT) mice but lose more weight and have increased mortality. WT or ICOS ${ }^{-1-}$ mice were challenged intratracheally with $1.0 \mathrm{U} \mathrm{kg}^{-1}$ bleomycin. (a) ICOS ${ }^{-1-}$ mice had increased mortality and (b) lost more weight. (c) Weight loss in ICOS ${ }^{-1-}$ and WT mice that survived until day 18 after challenge. Survival $P$ value shown from log-rank test. Weight loss was normalized to each mouse's starting weight on day 0 and $P$ values were determined using a mixed-effects linear regression model to account for missing values from mortality. Shown are the indicated $N$ per group from two independent experiments combined. (d) Representative histological sections from the left lung were stained with trichrome to show similar collagen deposits and cell infiltration in surviving WT or ICOS ${ }^{-1-}$ mice. (e) On day 18 of the experiment, surviving mice were killed and right lung was removed for hydroxyproline assay as a surrogate for collagen deposition. Dotted line indicates mean hydroxyproline level detected in untreated WT mice. Unpaired Student's $t$-test was used to determine significance. (f) Representative hematoxylin and eosin-stained tissue sections from WT or ICOS ${ }^{-1}$ - mice on day 5 post-bleomycin challenge. Left lungs were scored for (g) edema by the presence of red blood cells in airways or (h) inflammation by the presence of leukocytic infiltration. Sections were blindly scored based on the overall area of the lung exhibiting edema or inflammation and the degree to which cell influx was observed. (i) Total protein measured in day 5 bronchoalveolar lavage. (j) Lung permeability was determined by administering fluorescein isothiocyanate (FITC)-dextran intratracheally and measuring FITC in the serum $1 \mathrm{~h}$ later. $P$ value is two-way analysis of variance comparing the genotypes with 4-5 mice per group per time point. Dotted line indicates the average FITC measurement in serum from phosphate-buffered saline-treated control mice. (k) Lung neutrophils were identified by flow cytometry as live $\mathrm{CD} 11 \mathrm{~b}^{+} \mathrm{Ly}_{6 \mathrm{G}}{ }^{+}$cells in total digested lung tissue. KO, knockout; NS, not significant.

$\left.\mathrm{ICOS}^{+}\right)$or $\mathrm{ICOS}^{+}$iNKTs $\left(\mathrm{CD} 3{ }^{+} \mathrm{CD}^{+} 6^{+} \mathrm{ICOS}^{+}\right)$between IPF lungs and the non-fibrotic donor lungs and found that explanted IPF lungs contained lower percentages of ICOS ${ }^{+}$ ILCs compared with control lungs but comparable percentages

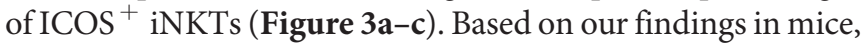
it is possible that the decrease in ICOS-expressing innate cells in the IPF lungs may have a role in the recovery of IPF patients from lung injury during acute exacerbations.
IL-5, produced primarily by ILC2s, is reduced in ICOS ${ }^{-I-}$ mice at day 3 post-bleomycin challenge

To determine the function of $\mathrm{ICOS}^{+}$cells in protection from lung injury-associated death, we examined their cytokine profiles after bleomycin challenge. We found that day 3 IL-5 expression in the lungs of mice was dependent on ICOS, although IL-13 was unaffected (Figure 4a,b). The majority of IL-5-producing lung cells were ILC2s, rather than iNKTs or 
a Day 3

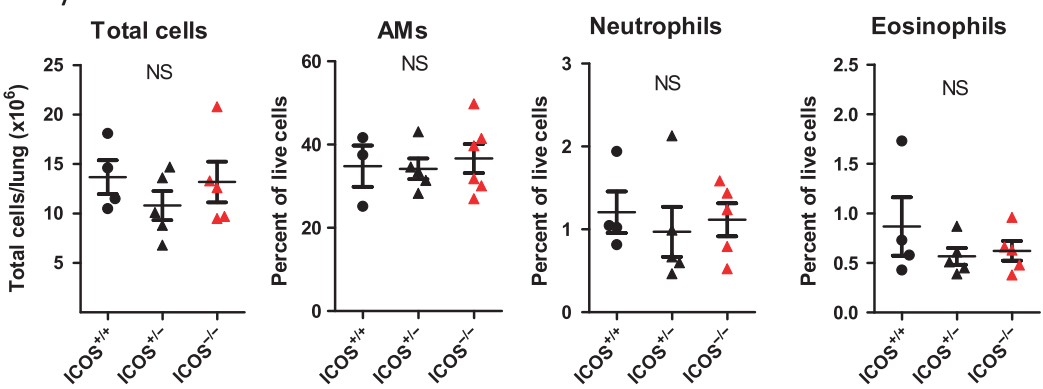

b Day 3
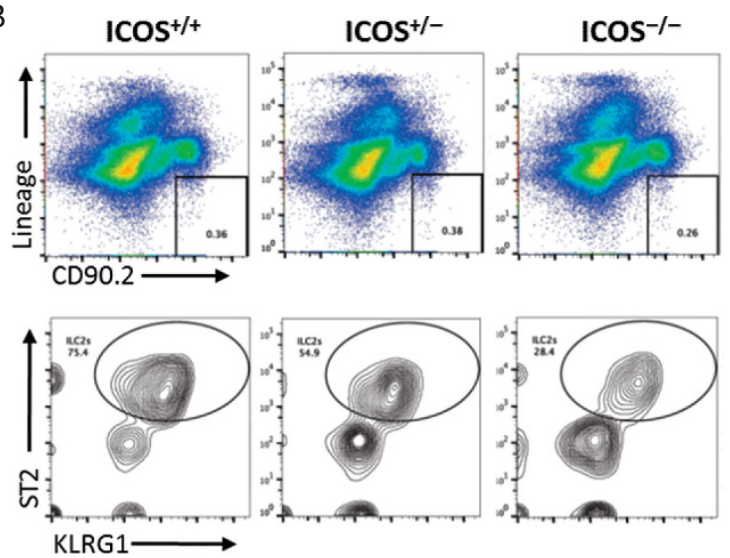

C

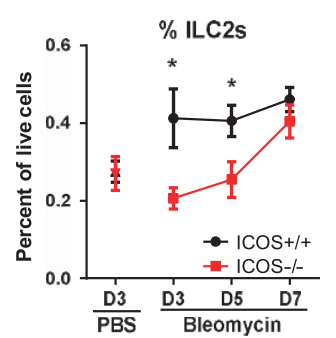

d

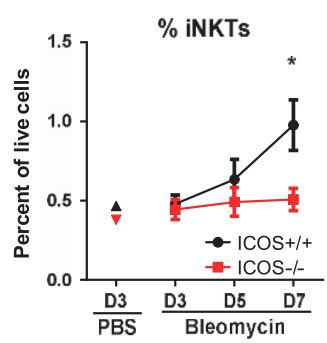

e

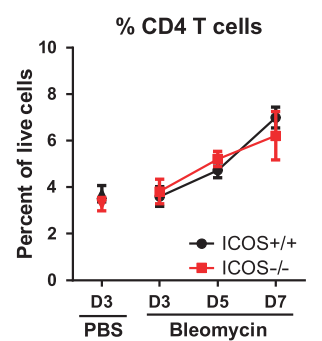

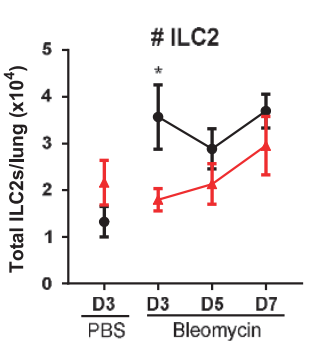
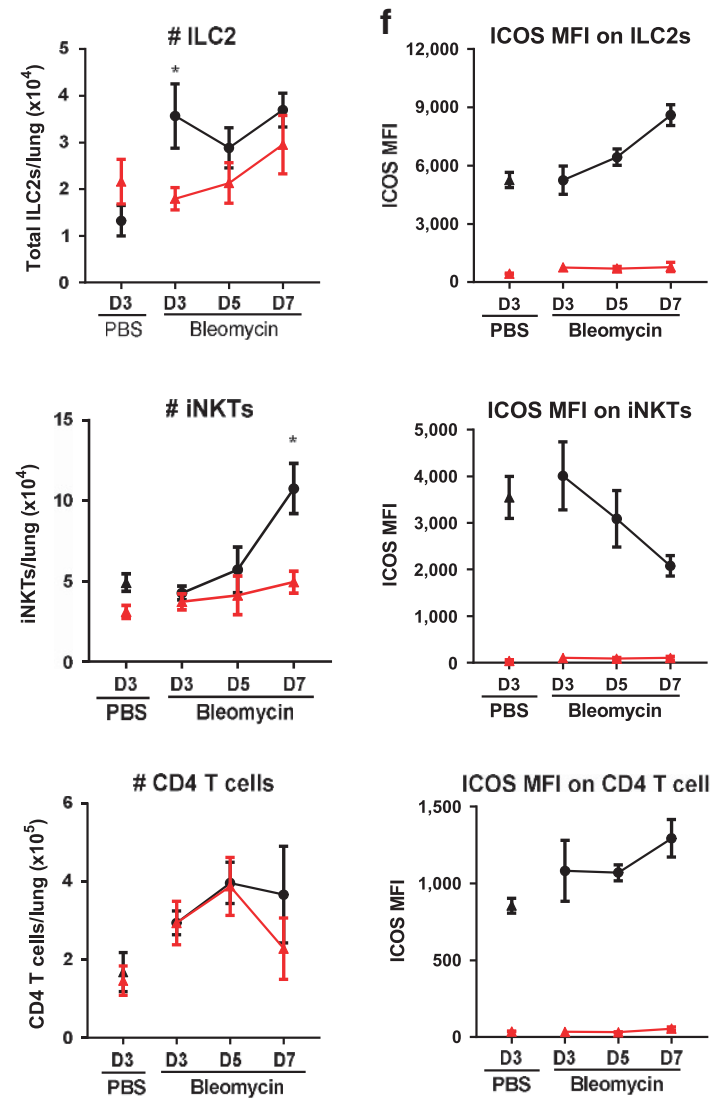

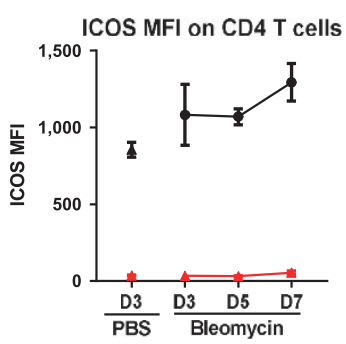


CD4 T cells (Figure 4c). Total IL-13 + cell percentages and numbers were not reduced in ICOS $^{-1-}$ mice (Figure $4 \mathbf{b}, \mathbf{d}$ ). Although the ILC2 did produce IL-13, they were not the major source of IL-13 + cells in the lungs. Thus the decrease in ILC2s did not affect the total number of lung IL-13 + -producing cells after bleomycin challenge. Interestingly, the loss of ICOS did not affect the cell-intrinsic ability of ILC2s to produce IL-5 or IL-13 (Figure 4e), it only affected the absolute number of ILC2s. However, the overall decrease in ILC2 at day 3 (Figure 2c) led to reduced numbers of total IL-5 ${ }^{+}$ILC2s and IL-13 + ILC2s (Figure 4e,f). The defect in IL- ${ }^{+}$cells in the ICOS ${ }^{-1-}$ mice was gone by day 7 , suggesting that early, not late, ICOS-dependent IL-5 production was key to the protection from lung injury-induced death in the WT mice (see Supplementary Figure S7). ICOS-dependent variation in CD4 $\mathrm{T}$ cell cytokines was observed at day 7 , but these differences were independent of bleomycin challenge (see Supplementary Figure S8 ). Thus ICOS ${ }^{-/}$mice produce less IL-5 early after bleomycin challenge primarily due to loss of total ILC2s.

\section{Treatment with exogenous IL-5 protects both $\mathrm{ICOS}^{-I-}$ and WT mice from bleomycin-induced mortality}

As IL-5-producing ILC2 expansion was associated with protection from lung injury, we speculated that IL-5 could be a protective effector cytokine during the early response. Strikingly, when ICOS $^{-1-}$ mice were treated with IL-5 at the

Table 1 Characteristics of study subjects ${ }^{a}$

\begin{tabular}{|c|c|c|c|}
\hline Characteristic & IPF $(n=9)$ & Control $(n=13)$ & $\boldsymbol{P}$-value \\
\hline Age, mean (s.d.) & $58.7(7.6)$ & $58.0(4.1)$ & 0.782 \\
\hline Male, $n(\%)$ & $6(67)$ & $4(31)$ & 0.192 \\
\hline \multicolumn{4}{|l|}{ Race/ethnicity } \\
\hline White, $n(\%)$ & $6(67)$ & $8(62)$ & 1.000 \\
\hline Non-White, $n$ (\%) & $3(33)$ & $5(37)$ & 1.000 \\
\hline Ever smoker, n (\%) & $6(67)$ & $7(54)$ & 0.674 \\
\hline Pack years, mean (s.d.) & $28.2(25.6)$ & $16.9(12.9)$ & 0.325 \\
\hline $\begin{array}{l}\text { Immunosuppressive } \\
\text { therapy, } n(\%)\end{array}$ & $2(22)$ & $2(15)$ & 1.000 \\
\hline Diabetes, $n$ (\%) & $1(11)$ & $4(31)$ & 0.360 \\
\hline
\end{tabular}
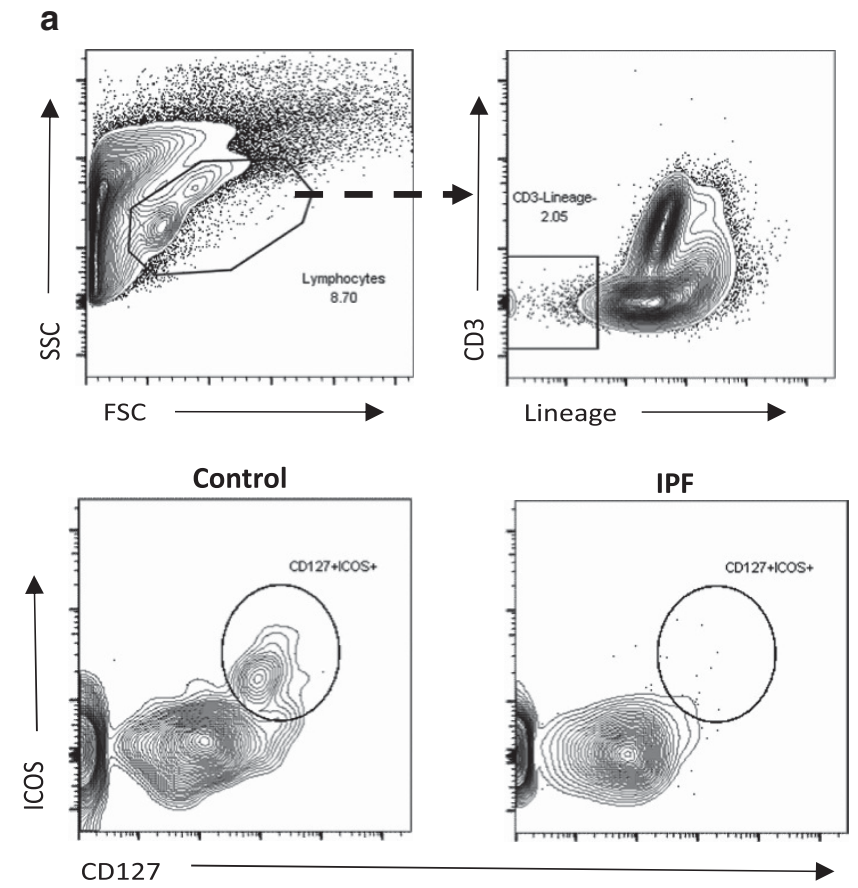

b

b $\operatorname{ICOS}^{+} \mathrm{ILCs}$

C $\operatorname{ICOS}^{+} \mathrm{NKTS}$
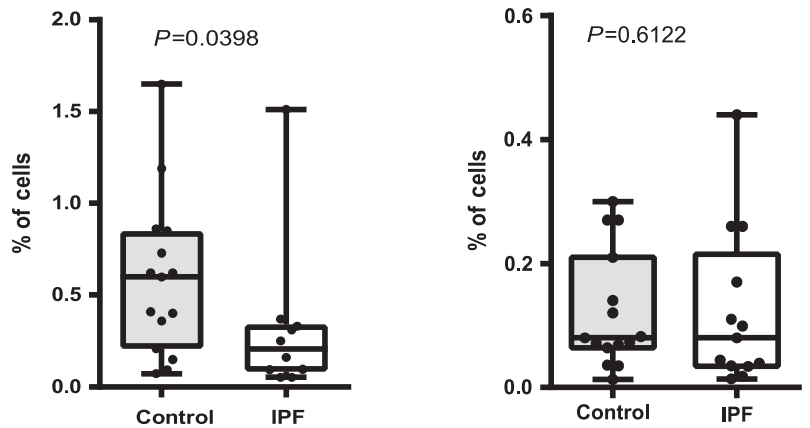

Figure 3 Lower percentage of ICOS + innate lymphocytes (ILCs) are found in the lungs of idiopathic pulmonary fibrosis (IPF) patients. (a) Gating strategy to identify ICOS ${ }^{+}$ILCs in human lung tissue from IPF patients or controls. For IPF patients, tissue was taken from the diseased lung at the time of lung transplant, and for controls, lung tissue was taken from deceased organ donors with no history of lung disease or fibrosis. Lymphocytes were gated on forward and side scatter (FSC and SSC, respectively) for size (left top panel), followed by a lineage-negative selection (right top panel). Of these cells, ICOS ${ }^{+}$ILCs were identified as ICOS ${ }^{+} \mathrm{CD}_{127^{+}}$. Shown are a representative control lung (left bottom panel) and a representative IPF lung (right bottom panel). Enumeration of (b) ILCs or (c) invariant natural killer T cells (NKTs), defined as $\mathrm{CD}^{+}{ }^{+} \mathrm{CD}_{5}{ }^{+}{ }^{+} \mathrm{COS}{ }^{+}$, in the IPF and control lungs. Shown is the proportion of each cell type out of total isolated lung leukocytes, with significance determined by Mann-Whitney non-parametric test.

Figure 2 Increased early inflammation in ICOS ${ }^{-1-}$ mice is associated with failed expansion of type 2 innate lymphocytes (ILC2s) and invariant natural killer T cells (iNKTs) but not CD4 T cells. (a) Total cells recovered from the lungs on day 3, and the proportion identified as alveolar macrophages (AMs; CD11 $\mathrm{c}^{+}$Siglec- $\left.\mathrm{F}^{+}\right)$, neutrophils $\left(\mathrm{CD} 11 \mathrm{~b}^{+} \mathrm{Ly}_{6 \mathrm{G}}{ }^{+}\right)$, and eosinophils $\left(\right.$Siglec- $\left.\mathrm{F}^{+} \mathrm{CD} 11 \mathrm{c}^{\mathrm{lo}}\right)$. No significant differences (NS) were found by analysis of variance with Tukey's posttest. (b) ILC2s were identified as Lineage ${ }^{-}$Thy $1.2^{+} \mathrm{ST} 2^{+} \mathrm{KLRG} 1^{+/}$. Representative flow plots of ILC2 proportions in the lungs are shown at 3 days post-bleomycin challenge. Proportion and total number of $(\mathbf{c})$ ILC2s, (d) iNKTs $\left(C D 3^{+}\right.$CD1d Tetramer $\left.^{+}\right)$, and $(\mathbf{e})$ CD4 T cells $\left(\mathrm{CD}^{+} \mathrm{CD}^{+}\right)$are shown for ICOS ${ }^{-1-}$ and wild-type mice over days 3-7. Shown is mean and s.e.m. with $N=6-15$ mice per time point per group. $P$ value determined by unpaired Student's $t$-test comparing ICOS ${ }^{+/+}$to ICOS ${ }^{-1-}$ mice at each time point and significance $<0.05$ is indicated by asterisk $\left({ }^{*}\right)$. (f) Expression of ICOS was measured by mean fluorescent intensity (MFI) using flow cytometry on the indicated cell types. 

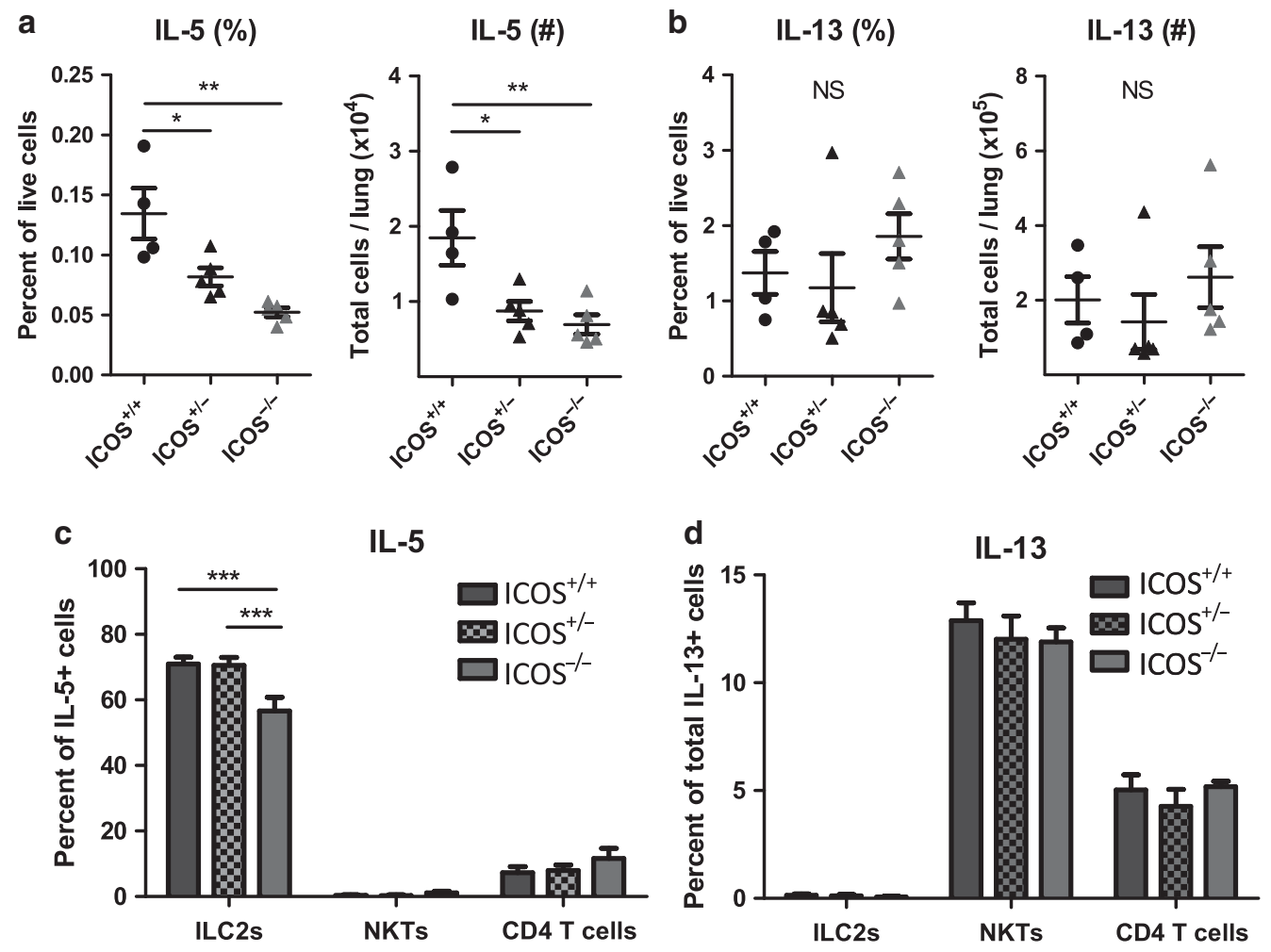

e IL-5 $5^{+}$ILC2S (\%)
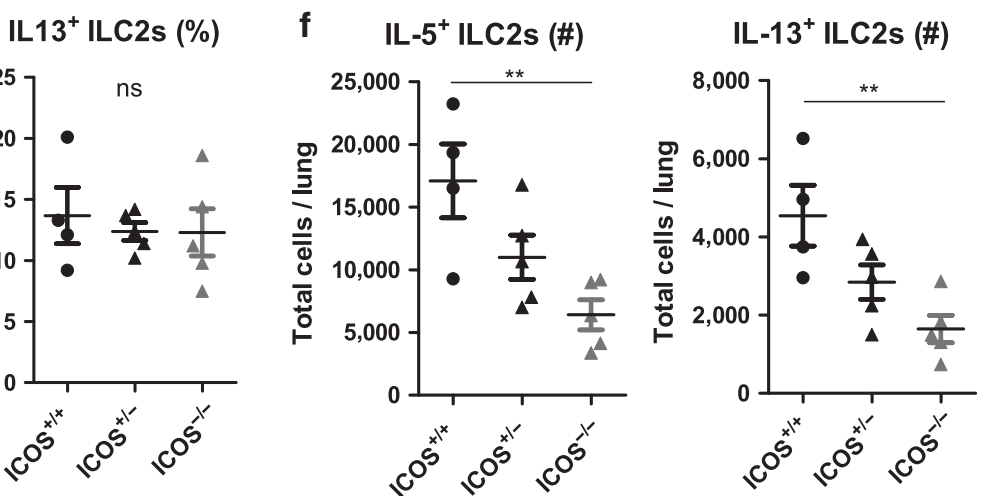

Figure 4 Type 2 innate lymphocyte (ILC2) cells are the major producer of interleukin (IL)-5 3 days after bleomycin challenge. The percentage and total number of IL-5-producing (a) or IL-13-producing cells (b) was determined 3 days after bleomycin challenge. Significant differences were determined by analysis of variance (ANOVA) with asterisk indicating ${ }^{*} P<0.05$ or ${ }^{* *} P<0.01$ by Tukey's posttest. Shown is one representative of three independent experiments. (c) IL-5-producing and (d) IL-13-producing cells were identified as ILC2s, invariant natural killer T cells (NKTs), or CD4 T cells and shown as the percentage of each cell type out of total IL- $5^{+}$or IL-13 + lung cells. Significant differences were determined by ANOVA with asterisks indicating ${ }^{* * \star} P<0.001$ by Tukey's posttest. (e) The percentage of ILC2s producing IL-5 ${ }^{+}$or IL-13 + was similar between strains; (f) however, differences were found in total IL-5 $5^{+}$and IL-13+ ILC2s owing to the overall decrease in ILC2 numbers. NS, not significant.

time of bleomycin challenge, they were protected from death despite similar weight loss (Figure 5a). Protection against death was lost when treatment was started $24 \mathrm{~h}$ after bleomycin challenge (Figure $\mathbf{5 b}$ ). These data indicate that IL-5 alone is sufficient to protect ICOS ${ }^{-1-}$ mice from lung injury-induced death when treated at the time of injury. Although our findings in ICOS ${ }^{-1-}$ mice were dramatic, lung injury in humans occurs in patients who express ICOS. To determine the therapeutic potential of IL- 5 to treat lung injury, we challenged WT mice with a higher dose of bleomycin that induced $80 \%$ mortality.
Surprisingly, WT treated with IL-5 at the time of bleomycin challenge had only $20 \%$ mortality that occurred at a later time point (Figure $5 \mathbf{c}$ ). Treatment starting $24 \mathrm{~h}$ post-bleomycin challenge had no effect on survival (Figure 5d). We found that IL-5 treatment caused a transient increase in total cells, including eosinophils, in the BAL at day 3 post-bleomycin, but by day 5 the BAL numbers and eosinophilia was similar in both groups (Figure 6a,b). Importantly, mice receiving IL-5 treatment had reduced RBCs in the airways indicating a reduction in pulmonary edema (Figure $\mathbf{6 c}-\mathbf{e}$ ), suggesting that 
a

\section{IL-5 Treatment day 0}
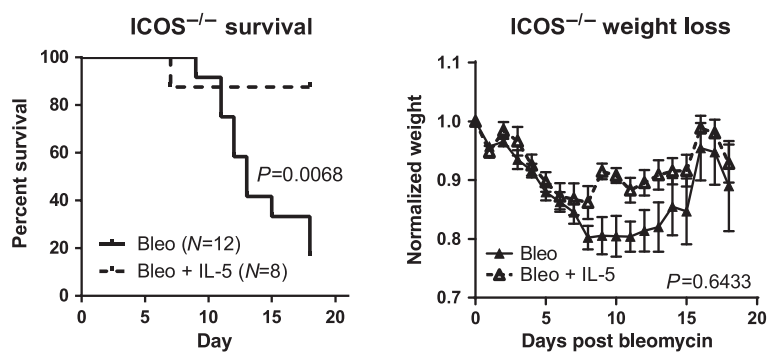

b

\section{IL-5 Treatment day 1}
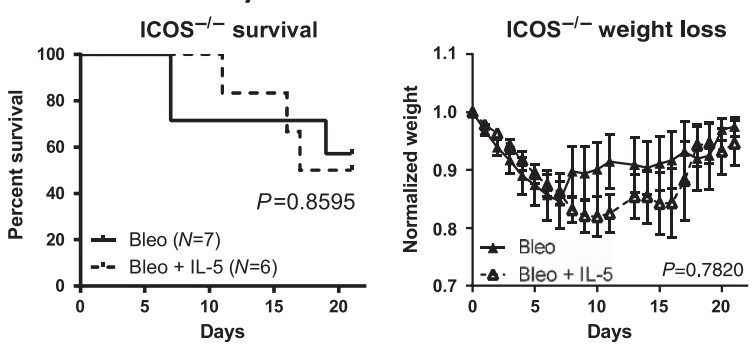

C

IL-5 Treatment day 0
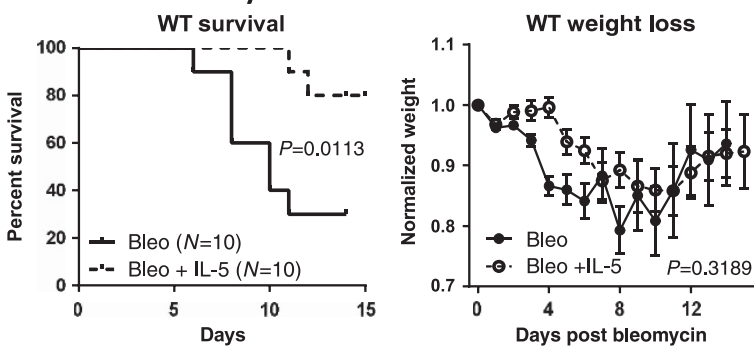

d

IL-5 Treatment day 1
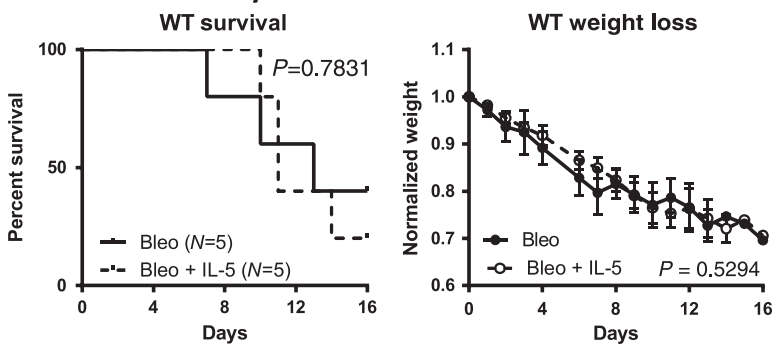

Figure 5 Treatment with recombinant interleukin (IL)-5 improves survival from bleomycin (Bleo)-induced lung injury. ICOS ${ }^{-1-}$ mice were treated with $1.0 \mathrm{U} \mathrm{kg}^{-1}$ bleomycin with or without recombinant IL-5 (50 ng per mouse) concurrent with (a) challenge or (b) 1 day after challenge. Survival and weight loss were measured. Wild-type (WT) C57BI/6 mice were treated with a two-fold higher dose of bleomycin $\left(2.0 \mathrm{U} \mathrm{kg}^{-1}\right)$ with or without recombinant IL-5 (50 ng per mouse) on (c) day 0 or (d) day 1 after challenge. Survival curve $P$ values determined by log-rank test.

IL- 5 acts to suppress early vascular leakage and reduce the effects of bleomycin-induced lung injury. In both $\mathrm{ICOS}^{-1-}$ and WT mice, IL-5 treatment had no effect of the number of Tregs recruited to the lungs on day 7 (see Supplementary Figure S9). These data suggest that IL-5 has potential therapeutic effects in bleomycin-induced lung injury independent of the presence of functional ILC2s, Tregs, or endogenous IL-5 production.

\section{DISCUSSION}

Our study demonstrates that early innate type 2 immune responses, driven by cells expressing ICOS, protect against lung injury-associated death. We found that $\operatorname{ICOS}^{-1-}$ mice have increased mortality in two models of lung injury. Further, they had increased edema and lung permeability as early as 5 days postchallenge, suggesting that ICOS expression is important for maintaining barrier function in the lungs. $\mathrm{IL}-5^{+}$ILC2s expanded quickly after lung injury in WT mice but not in $\mathrm{ICOS}^{-1-}$ leading to an overall defect in IL-5 ${ }^{+}$cells in the lungs. We now demonstrate that IL-5 is a critical ILC2 product that protects mice from lung injury-associated death. In fact, IL-5 was sufficient to improve survival in WT mice where ICOS signaling and ILC2 responses are intact. IPF patients also had fewer ICOS $^{+}$ILCs in their lungs at the time of transplant, suggesting that $\mathrm{ICOS}^{+}$innate lung cells may impact human disease as well.

Our mouse studies revealed that the protective role of ICOS is driven not by attenuation of fibrosis but by protection during the lung injury phase. Although the type 2 response may have pathogenic effects in lung fibrosis, mostly through IL-13, ${ }^{16,17}$ activation of the type 2 pathway is also critical for effective tissue repair after lung damage. Previous studies have shown ILC2s produce tissue-reparative factors, such as amphiregulin, ${ }^{18,19}$ IL- $4,{ }^{20}$ and IL-13, but interestingly, we found ILC2s produced little of the total IL-13 measured in the bleomycin model. Our findings now argue for a novel role for IL-5, produced primarily by ILC2s, in preventing lung injury or mediating repair. In fact, our data that (1) IL-5 treatment must start concurrent with the lung injury, and (2) IL-5 reduced RBC influx into the airways, leads us to speculate that prevention of lung injury is the primary function of IL-5 in this model. The mechanisms by which IL-5 protects from lung injury remain unknown.

The IL-5 receptor is expressed on eosinophils, B1 B cells, monocytes, and neutrophils. ${ }^{21-23}$ Eosinophils in particular have been shown to have immunoregulatory function and to produce anti-inflammatory cytokines, including IL-10 and transforming growth factor- $\beta$, and the immunoregulatory molecule indoleamine 2,3-dioxygenase. ${ }^{24-26}$ Although eosinophils have been shown to be increased in the IPF lungs, IL-5 levels were not elevated in BAL fluid. ${ }^{27,28}$ Eosinophils can be either tissue resident with anti-inflammatory function or recruited during inflammation and have pro-inflammatory function. ${ }^{29}$ We expect that IL-5 may act on eosinophils as well as other cell types shown to express the IL- 5 receptor, such as neutrophils and monocytes. A role of IL-5 on monocytes was recently reported in sepsis, and IL-5 was able to protect mice from cecal-ligation-induced sepsis in an eosinophil-independent manner. ${ }^{22}$ Therefore, monocytes are also a potential target of IL-5 that may mediate protection from lung injuryassociated death. Neutrophils have been shown to be a major player in lung injury; however, how IL-5 may affect these cells 


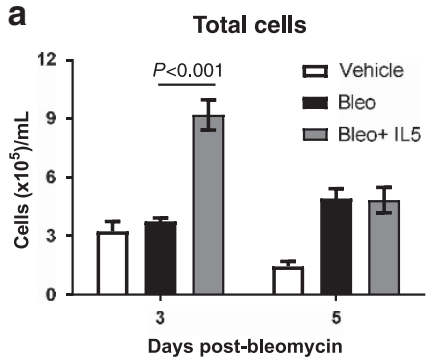

C

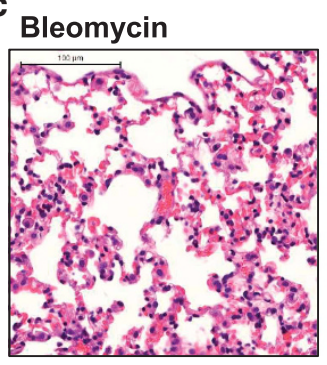

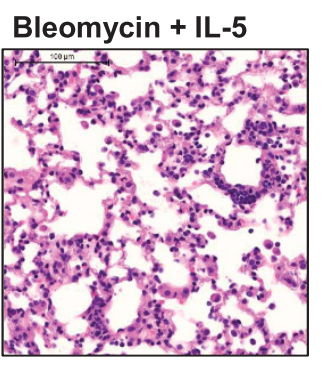

b

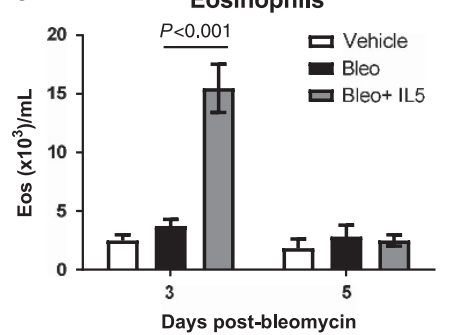

d

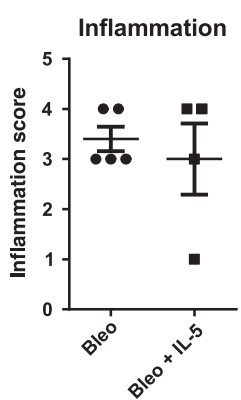

e

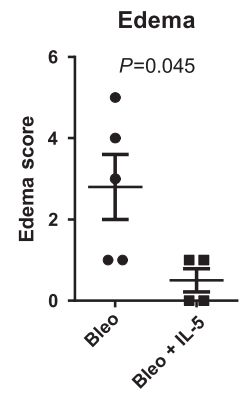

Figure 6 Interleukin (IL)-5 treatment results in increased eosinophils in the bronchoalveolar lavage (BAL) and reduced red blood cells in the airways. $\mathrm{ICOS}^{-1-}$ mice were challenged with bleomycin and treated with IL-5 or vehicle control. (a) Total cells and (b) eosinophils $\left(\mathrm{Ly} 6 \mathrm{G}^{-}\right.$Siglec- $\left.\mathrm{F}^{\text {hi }} \mathrm{CD} 11 \mathrm{c}^{\mathrm{low}}\right)$ were measured in the BAL on days 3 and 5 . Significance determined by analysis of variance with Tukey's posttest with 3-4 mice per group per time point. (c) Representative histology from ICOS ${ }^{-1}$ mice challenged with bleomycin (left panel) or bleomycin with IL-5 (right panel). (d) Inflammation and (e) edema was scored as described in Figure 1, and significance determine by unpaired $t$-test.

remains unstudied. Additionally, it is possible that IL-5 could act directly on structural cells in the lungs, such as epithelium or endothelium, to reduce permeability. Understanding the mechanisms by which IL-5 protects against lung injuryinduced mortality in mice will provide essential knowledge about how the immune system safeguards lungs against irreversible damage.

The role of lung injury in IPF is not completely understood, but repeated damage to pulmonary epithelium has been implicated. ${ }^{15}$ Acute exacerbations are a major cause of morbidity and mortality of IPF patients. Although acute exacerbations often occur without identifiable cause, lung injury has been proposed to be a factor in the decline of IPF patients after acute exacerbations. ${ }^{30,31}$ Interestingly, corticosteroid treatment in IPF patients during acute exacerbations contributed to increased mortality, and a clinical trial using immune suppressants in patients was terminated owing to worse outcomes in the treatment group. ${ }^{4,5}$ These trials, in combination with our current data, suggest that IPF patients may benefit from increased immune activation through an ICOS-dependent pathway or therapeutic use of IL-5. In our model, one treatment with IL-5 at the time of injury was sufficient for protection, suggesting that IL-5 has lasting downstream effects. We expect that IL-5 treatment would be a benefit during acute exacerbations, when pulmonary function is reduced owing to a lung injury event.

IL-5 treatment may broadly benefit recovery from other types of lung injury. A variety of factors, including respiratory infections and sepsis, can result in lung injury that leads to Acute Respiratory Distress Syndrome (ARDS). ARDS is characterized by lung edema and hypoxemia and currently has no specific therapy. ${ }^{32}$ Bleomycin is widely used as a model of ARDS, and therefore our findings may also be applicable to ARDS and related syndromes. ${ }^{33}$ Our data that ICOS $^{-1-}$ mice die early during influenza infections supports the hypothesis that ICOS deficiency is a risk factor for lung injury-related mortality. Interestingly, ILC2s expand during early infection with influenza and are associated with viral clearance and recovery. ${ }^{18,34}$ Thus our findings may have important implications for the treatment of ARDS and other forms of acute lung injury.

\section{METHODS}

Human lung procurement from donors and IPF patients. Lung tissue specimens were collected from nine IPF patients who underwent lung transplantation at the University of Chicago from August 2013 to May 2015. IPF diagnosis was established by a multidisciplinary group of pulmonologists and dedicated chest pathologists, and radiologists in concordance with the American Thoracic Society/European Respiratory Society criteria, and in agreement with recent guidelines. ${ }^{30}$ IPF patients were excluded at study entry if they had clinical or serological markers of an autoimmune disease, including rheumatoid arthritis, malignancies, active infection, and drug exposure or occupational exposures known to cause interstitial lung disease. The study is approved by the University of Chicago Institutional Review Board and all transplant patients signed informed consent. All IPF patients had severe hypoxemia but were stable, uninfected, and called from home to receive their transplant surgery. Control lungs were obtained through the Gift of Hope Regional Organ Bank of Illinois and processed within $48 \mathrm{~h}$ of organ clamp time. The University of Chicago Institutional Review Board has determined that use of human donor lungs that could not be transplanted does not constitute human 
subjects research and is exempt from Institutional Review Board oversight.

Isolation of human lung leukocytes. To obtain lung leukocytes from IPF or control lungs, right lower lobes were perfused with $150-200 \mathrm{ml}$ sterile $2 \%$ fetal bovine serum/phosphate-buffered saline (PBS) using a $50 \mathrm{ml}$ syringe. Tissue was minced using dissection scissors to an approximate size of $2 \mathrm{~mm}^{3}$. Minced tissue was digested in RPMI media containing $10 \%$ fetal bovine serum, 120 Units ml $^{-1}$ Collagenase D (Sigma-Aldrich, Darmstadt, Germany), and $2 \mu \mathrm{g} \mathrm{ml}^{-1}$ DNase I (Worthington Biochemical Corporation, Lakewood, NJ) at $37^{\circ} \mathrm{C}$ for $90 \mathrm{~m}$. Mononuclear cells were enriched by gradient centrifugation using Histopaque-1077 (Gibco Laboratories, Gaithersburg, MD). Isolated leukocytes from IPF and control lungs were cryopreserved until analysis.

Mice. C57BL/6 mice (WT) were purchased from Harlan Laboratories (Indianapolis, IN). ICOS ${ }^{-1-}\left(\mathrm{B}^{-\mathrm{ICOS}^{-1-}}\right)$ mice were a generous gift of Dr Flavell. ${ }^{35} \mathrm{ICOS}^{+1+}, \mathrm{ICOS}^{+1-}$, and $\mathrm{ICOS}^{-1-}$ littermates were generated from WT $\times$ B6.ICOS ${ }^{-1-}$ parents. All animal procedures and housing were approved by the University of Chicago Animal Resources Center. The studies conformed to the principles set forth by the Animal Welfare Act and the National Institutes of Health guidelines for the care and use of animals in biomedical research.

Cellular analysis and flow cytometry. Perfused mouse lungs were dissociated by mechanical mincing followed by digestion with $150 \mathrm{U} \mathrm{ml}^{-1}$ Collagenase D (Gibco) in $10 \mathrm{ml}$ Dulbecco's modified Eagle's medium for $1 \mathrm{~h}$. Samples were then washed and counted. For phenotyping, $5 \times 10^{5}$ cells were suspended in $50 \mu \mathrm{l}$ of buffer (PBS containing $0.1 \%$ sodium azide and $0.2 \%$ bovine serum albumin) and blocked using 2.4G2 (anti-CD16/32) and then stained with surface antibodies. To measure intracellular cytokine production, $1 \times 10^{6}$ lung cells per mouse were stimulated with phorbol myristate acetate $\left(10 \mathrm{ng} \mathrm{ml}^{-1}\right)$ and ionomycin (500 $\mathrm{ng} \mathrm{ml}^{-1}$ ) for $30 \mathrm{~min}$ followed by Brefeldin A $\left(1 \mu \mathrm{g} \mathrm{ml}^{-1}\right)$ for an additional $4-5 \mathrm{~h}$ prior to staining. Isotype controls or no Brefeldin A controls were used for all cytokine staining. For intracellular staining, cells were fixed with $1 \%$ paraformaldehyde and permeabilized with $0.5 \%$ saponin in PBS. Flow antibodies were purchased from Biolegend (San Diego, CA), eBioscience (San Diego, CA), and BD Biosciences (San Jose, CA), and iNKTs were identified using phycoerythrin-conjugated $\mathrm{CD} 1 \mathrm{~d}^{\mathrm{PBS} 57}$ tetramers (National Institutes of Health Tetramer Facility at Emory University). Flow cytometric analysis was performed on an LSRFortessa or LSRII (BD Biosciences), and the data were analyed with the FlowJo software (Tree Star, Ashland, OR). All instruments are maintained by the University of Chicago Flow Cytometry and Antibody Technology Core Facility.

Bleomycin model. Bleomycin for Injection USP (15 Units per vial from Teva Pharmaceuticals USA, Sellersville, PA, USA) was reconstituted at 3 Units $\mathrm{ml}^{-1}$ in PBS and stored at $-80^{\circ} \mathrm{C}$ until use. Stock solution was diluted in additional PBS for intratracheal instillations (i.t.). Mice were weighed 1 day prior to intratracheal instillations to determine appropriate unit $\mathrm{kg}^{-1}$ dose. Recombinant murine IL-5 (50 ng per mouse, Biolegend) was added to calculated bleomycin doses prior to intratracheal instillation. Lung collagen content was quantified by hydroxyproline assay and trichrome staining of formalin-fixed tissue sections. Lung inflammation was determined by hematoxylin and eosin staining of lung tissue sections or flow cytometry. Lung edema was scored as follows for one lung lobe per mouse: 0 , no RBCs present; 1 , small patch of airway with RBCs infiltrating; $2,1 / 8$ of lung lobe contains RBCs; 3, 1/4 of lung lobe contains RBCs; 4, 1/2 of lobe contains RBCs; and 5, entire lobe shows RBCs in airways. For all histology scoring, the scorer was blinded to the mouse genotype and/or treatment, and slides were presented in a random order. The same lung lobe was scored for each mouse. Lung permeability was determined by administering fluorescein isothiocyanate-dextran $70\left(10 \mathrm{mg} \mathrm{kg}^{-1}\right.$, Sigma) intratracheally and measuring fluorescein isothiocyanate in the serum $1 \mathrm{~h}$ later. $^{36}$ Total protein in BAL was determined by bicinchoninic acid assay (Sigma).

Statistical analysis. Unless specified in figure legends, statistical analyses were performed with the GraphPad Prism software (San Diego, CA), and a $P$ value $<0.05$ was considered significant $\left({ }^{\star} P<0.05\right.$; ${ }^{* *} P<0.01 ;{ }^{* *} P<0.001 ; \mathrm{NS}=$ not significant). Experiments with two groups were analyzed using an unpaired Student's two-tailed $t$-test. Experiments with greater than two groups were analyzed with a oneway analysis of variance and post hoc Tukey's test. Error bars represent the s.e.m.

SUPPLEMENTARY MATERIAL is linked to the online version of the paper at http://www.nature.com/mi

\section{ACKNOWLEDGMENTS}

We thank the IPF patients and families who generously agreed to participate in this study, the University of Chicago IPF transplant team and surgeons, especially Dr Edward Garrity, Dr Chris Wigfield, Dr Wickii Vigneswaran, and Dr Takeyoshi Ota. For facilitating the Gift of Hope control lung donations, we thank the Lung Biospeciman Core, especially Dr Julian Solway and Dr Steve White. We also thank Dr Amanda Marzo for her assistance with the murine influenza model. This work was supported by R21 Al126031; the University of Chicago Institute for Translational Medicine (ITM) Clinical and Translational Science Award UL1 RR024999; the IPFnet Cowlin Fellowship Award to S.T.M.; T32 HL007605 to C.L.H., S.T.M., C.A.B. and A.O.A.; 1R25 HL096383; and a supplement to U19 AI095230 to J.C. The University of Chicago Flow Cytometry Core is supported by P30 CA014599.

\section{AUTHOR CONTRIBUTIONS}

Conception and design: C.L.H., S.T.M., C.A.B. and A.I.S. Data acquisition, analysis, and interpretation: C.L.H., S.T.M., D.B., J.C., M.R.J., K.M.B., K.A.M.M., P.A.V., A.O.A, J.W.W., M.Y.T., T.V.M., M.E.S., I.N. and A.I.S. Drafting the manuscript: C.L.H., C.A.B., K.M.B. and A.I.S. All authors had substantial contributions to critically revising this manuscript for important intellectual content and have provided their approval.

\section{DISCLOSURE}

The authors declared no conflict of interest.

c) 2018 Society for Mucosal Immunology

\section{REFERENCES}

1. Borensztajn, K., Crestani, B. \& Kolb, M. Idiopathic pulmonary fibrosis: from epithelial injury to biomarkers-insights from the bench side. Respiration $\mathbf{8 6}$, 441-452 (2013).

2. Ahluwalia, N., Shea, B.S. \& Tager, A.M. New therapeutic targets in idiopathic pulmonary fibrosis. Aiming to rein in runaway wound-healing responses. Am. J. Respir. Crit. Care Med. 190, 867-878 (2014).

3. Zoz, D.F., Lawson, W.E. \& Blackwell, T.S. Idiopathic pulmonary fibrosis: a disorder of epithelial cell dysfunction. Am. J. Med. Sci. 341, 435-438 (2011).

4. Wells, A.U., Behr, J., Costabel, U., Cottin, V. \& Poletti, V. Triple therapy in idiopathic pulmonary fibrosis: an alarming press release. Eur. Respir. J. 39, 805-806 (2012).

5. Papiris, S.A. et al. Survival in Idiopathic pulmonary fibrosis acute exacerbations: the non-steroid approach. BMC Pulm. Med. 15, 162 (2015).

6. Adengunsoye, A.H. et al. Skewed lung CCR4 to CCR6 CD4 + T cell ratio in idiopathic pulmonary fibrosis is associated with pulmonary function. Front. Immunol. 7, 516 (2016).

7. Herazo-Maya, J.D. et al. Peripheral blood mononuclear cell gene expression profiles predict poor outcome in idiopathic pulmonary fibrosis. Sci. Transl. Med. 5, 205ra136 (2013).

8. Rudd, C.E. \& Schneider, H. Unifying concepts in CD28, ICOS and CTLA4 co-receptor signalling. Nat. Rev. Immunol. 3, 544-556 (2003). 
9. Moore, T.V., Clay, B.S., Cannon, J.L., Histed, A., Shilling, R.A. \& Sperling, A.I. Inducible costimulator controls migration of T cells to the lungs via down-regulation of CCR7 and CD62L. Am. J. Respir. Cell Mol. Biol. 45, 843-850 (2011).

10. Tesciuba, A.G. et al. ICOS costimulation expands Th2 immunity by augmenting migration of lymphocytes to draining lymph nodes. J. Immunol. 181, 1019-1024 (2008).

11. Shilling, R.A. et al. Cutting edge: polymorphisms in the ICOS promoter region are associated with allergic sensitization and Th2 cytokine production. J. Immunol. 175, 2061-2065 (2005).

12. Maazi, H. et al. ICOS:ICOS-ligand interaction is required for type 2 innate lymphoid cell function, homeostasis, and induction of airway hyperreactivity. Immunity 42, 538-551 (2015).

13. Akbari, O. et al. ICOS/ICOSL interaction is required for $C D 4+$ invariant NKT cell function and homeostatic survival. J. Immunol. 180, 5448-5456 (2008).

14. Simpson, T.R., Quezada, S.A. \& Allison, J.P. Regulation of CD4 T cell activation and effector function by inducible costimulator (ICOS). Curr. Opin. Immunol. 22, 326-332 (2010).

15. Wynn, T.A. \& Ramalingam, T.R. Mechanisms of fibrosis: therapeutic translation for fibrotic disease. Nat. Med. 18, 1028-1040 (2012).

16. Gause, W.C., Wynn, T.A. \& Allen, J.E. Type 2 immunity and wound healing: evolutionary refinement of adaptive immunity by helminths. Nat. Rev. Immunol. 13, 607-614 (2013).

17. Firszt, R., Francisco, D., Church, T.D., Thomas, J.M., Ingram, J.L. \& Kraft, M. Interleukin-13 induces collagen type-1 expression through matrix metalloproteinase-2 and transforming growth factor-beta1 in airway fibroblasts in asthma. Eur. Respir. J. 43, 464-473 (2014).

18. Monticelli, L.A. et al. Innate lymphoid cells promote lung-tissue homeostasis after infection with influenza virus. Nat. Immunol. 12, 1045-1054 (2011).

19. Manzo, N.D., Foster, W.M. \& Stripp, B.R. Amphiregulin-dependent mucous cell metaplasia in a model of nonallergic lung injury. Am. J. Respir. Cell Mol. Biol. 47, 349-357 (2012).

20. Pelly, V.S. et al. IL-4-producing ILC2s are required for the differentiation of TH2 cells following Heligmosomoides polygyrus infection. Mucosal Immunol. 9, 1407-1417 (2016).

21. Gregory, B. et al. Differential regulation of human eosinophil IL-3, IL-5, and GM-CSF receptor alpha-chain expression by cytokines: IL-3, IL-5, and GM-CSF down-regulate IL-5 receptor alpha expression with loss of IL-5 responsiveness, but up-regulate $\mathrm{IL}-3$ receptor alpha expression. J. Immunol. 170, 5359-5366 (2003).

22. Linch, S.N., Danielson, E.T., Kelly, A.M., Tamakawa, R.A., Lee, J.J. \& Gold, J.A. Interleukin 5 is protective during sepsis in an eosinophilindependent manner. Am. J. Respir. Crit. Care Med. 186, 246-254 (2012).
23. Mesnil, C. et al. Lung-resident eosinophils represent a distinct regulatory eosinophil subset. J. Clin. Invest. 126, 3279-3295 (2016).

24. Spencer, L.A. et al. Human eosinophils constitutively express multiple Th1, Th2, and immunoregulatory cytokines that are secreted rapidly and differentially. J. Leukoc. Biol. 85, 117-123 (2009).

25. Odemuyiwa, S.O. et al. Cutting edge: human eosinophils regulate T cell subset selection through indoleamine 2,3-dioxygenase. J. Immunol. 173, 5909-5913 (2004).

26. Lee, J.J., Jacobsen, E.A., McGarry, M.P., Schleimer, R.P. \& Lee, N.A. Eosinophils in health and disease: the LIAR hypothesis. Clin. Exp. Allergy 40, 563-575 (2010).

27. Taniguchi, H. et al. Interleukin 5 and granulocyte-macrophage colonystimulating factor levels in bronchoalveolar lavage fluid in interstitial lung disease. Eur. Respir. J. 16, 959-964 (2000).

28. Boomars, K.A., Schweizer, R.C., Zanen, P., van den Bosch, J.M., Lammers, J.W. \& Koenderman, L. Eosinophil chemotactic activity in bronchoalveolar lavage from idiopathic pulmonary fibrosis is dependent on cytokine priming of eosinophils. Eur. Respir. J. 11, 1009-1014 (1998).

29. Mesnil, C. et al. Lung-resident eosinophils represent a distinct regulatory eosinophil subset. J. Clin. Invest. 126, 3279-3295 (2016).

30. Raghu, G. et al. An official ATS/ERS/JRS/ALAT statement: idiopathic pulmonary fibrosis: evidence-based guidelines for diagnosis and management. Am. J. Respir. Crit. Care Med. 183, 788-824 (2011).

31. Ghatol, A., Ruhl, A.P. \& Danoff, S.K. Exacerbations in idiopathic pulmonary fibrosis triggered by pulmonary and nonpulmonary surgery: a case series and comprehensive review of the literature. Lung 190, 373-380 (2012).

32. Ferguson, N.D. et al. The Berlin definition of ARDS: an expanded rationale, justification, and supplementary material. Intensive Care Med. 38, 1573-1582 (2012).

33. Aeffner, F., Bolon, B. \& Davis, I.C. Mouse models of acute respiratory distress syndrome: a review of analytical approaches, pathologic features, and common measurements. Toxicol. Pathol. 43, 1074-1092 (2015).

34. Gorski, S.A., Hahn, Y.S. \& Braciale, T.J. Group 2 innate lymphoid cell production of IL-5 is regulated by NKT cells during influenza virus infection. PLoS Pathog. 9, e1003615 (2013).

35. Dong, C. et al. ICOS co-stimulatory receptor is essential for T-cell activation and function. Nature 409, 97-101 (2001).

36. Chen, H., Wu, S., Lu, R., Zhang, Y.G., Zheng, Y. \& Sun, J. Pulmonary permeability assessed by fluorescent-labeled dextran instilled intranasally into mice with LPS-induced acute lung injury. PLOS ONE 9, e101925 (2014). 